\title{
Concepciones de Los PROFESORES Y estudiantes SOBRE prácticas de laboratorio del Proyecto Curricular de la LiCENCIATURA EN QuímicA DE LA Universidad Pedagógica Nacional.
}

\section{CONCEPTIONS OF THE PROFESSORS AND STUDENTS ON PRACTICAS of laboratory on the Curricualr Projecto of the Degree in Chemistry of the UPN.}

\author{
Luis Enrique Salcedo Torres* \\ salcedo@uni.pedagogica.edu.co \\ Julio César Rivera Rodríguez ** \\ jcrivera@uni.pedagogica.edu.co
}

\section{Abstract}

In this article the diagnostic made during first stage (CIUP appears $\tilde{A}$, $2002)^{1}$, on the conceptions and actions that professors and students pertaining to the cycle of fundamentation of the new curricular project of the degree in chemistry, maintain about practics of laboratory.

\section{RESUmen}

En este artículo se presenta el diagnóstico realizado durante la primera fase (CIUP - 2002) ${ }^{1}$, sobre las concepciones y acciones que profesores y estudiantes pertenecientes al ciclo de fundamentación del nuevo proyecto curricular de la licenciatura en química, mantienen acerca de las prácticas de laboratorio.

*Profesor Departamento de Química, Universidad Pedagógica Nacional. Programa Interinstitucional de Doctorado en Educación. Área Educación en Ciencias Naturales. (CIUP- DQU-014 -02) ${ }^{1}$

${ }^{* *}$ Coinvestigador. Estudiante de Maestría en Docencia de la Química. U.P.N. 


\section{INTRODUCCIÓN}

Las prácticas de laboratorio tal como son orientadas por los profesores y/o planteadas por los textos no familiarizan a los estudiantes con la metodología científica y no contribuyen al aprendizaje significativo de conceptos (Payá 1991). La visión del método científico que ellas generan sigue siendo demasiado simplista y conduce a creer que las teorías son simples conjeturas que los alumnos pueden elaborar después de breves períodos de trabajos de laboratorio y que pueden ser fácilmente contrastadas por medio de observaciones directas, aceptándose o rechazándose con base en experimentos aislados. Detrás de esta manera de orientar las prácticas de laboratorio, subyace en los profesores una concepción sobre la naturaleza de la metodología científica marcada por el inductivismo que infravalora la creatividad del trabajo científico, llevando a los estudiantes a pensar que la ciencia consiste en verdades absolutas, incontrovertibles y por consiguiente introduciendo rigidez e intolerancia por otras opciones. En tales casos, la evaluación de las mismas, se reduce a la manipulación de instrumentos o implementos de laboratorio y a la presentación de informes escritos, descuidando aspectos claves del trabajo científico tales como la identificación de problemas, el reconocimiento de variables, la emisión de hipótesis, el diseño de experimentos, el montaje experimental, la ejecución del experimento, la obtención de resultados, etc.

Si bien, la mayoría de trabajos en este campo han estado centrados en la educación secundaria, el problema se extiende al sector universitario con consecuencias aún más graves en la formación de profesionales que de una u otra manera requieren para el ejercicio de su profesión de un saber químico a profundidad. En este contexto, el problema para nosotros cobra importancia en los programas de formación de profesores de química quienes, como sabemos, serán los encargados de la enseñanza de dicha disciplina en la educación media y/o universitaria.

En nuestro país, se puede reconocer, en la enseñanza y el aprendizaje de las ciencias y de la química en particular, el amplio predominio en escuelas, colegios y universidades del paradigma de transmisión - asimilación, es decir, el predominio de la reproducción memorística de conocimientos, muy ligado a una concepción del mundo altamente empirista y a una concepción de la enseñanza y el aprendizaje de corte conductista (Salcedo y otros 1991). Hemos encontrado, por ejemplo, la prevalencia del aprendizaje memorístico sobre el aprendizaje procesual (Salcedo 1991), la permanencia de las ideas sobre la continuidad de la materia en alumnos futuros profesores de química (Salcedo y otros 1995), concepciones de los profeso- 
res de Química sobre las prácticas de laboratorio que no contribuyen al aprendizaje significativo de conceptos ni familiarizan al estudiante con el trabajo científico (Salcedo, 1994).

La Universidad Pedagógica a través de su Departamento de Química ofrece el programa de licenciatura para la formación de profesores de química; este programa ha sido planteado como un proyecto de investigación curricular que pretende la formación de un profesional con características definidas por el SER, el SABER y el SABER HACER. El reto de formación se da en la interfase entre la formación disciplinar y los núcleos del saber pedagógico; una formación científica en profundidad que conecte con sus relaciones pedagógicas y didácticas está en el centro de la propuesta formadora; así, el aporte que el trabajo experimental provee en este proceso es fundamental para el logro de los objetivos de formación del futuro educador en química.

En este contexto, hemos realizado la primera etapa del proyecto que nos ha permitido caracterizar el trabajo de laboratorio realizado por profesores y estudiantes dentro del nuevo proyecto curricular de licenciatura en química, a partir del cual se espera durante la segunda fase (año 2003), experimentar estrategias didácticas que favorezcan una toma de conciencia de esta problemática y contribuyan efectivamente a producir cambios en las concepciones y acciones que sobre prácti cas de laboratorio mantienen los profesores y en especial, para este proyecto, los que forman a futuros profesores de Química.

\section{REFERENTES CONCEPTUALES}

Las teorías actuales sobre como aprenden los estudiantes, han llevado a revisar la didáctica de las ciencias en general y de la química en particular, incluyendo el papel de las prácticas de laboratorio en el aprendizaje. Las contribuciones de epistemólogos de las ciencias y de las investigaciones sobre concepciones alternativas desarrolladas en los últimos veinte años, han aportado a la comprensión del papel que el experimento juega en la producción del conocimiento científico y al papel que tienen las prácticas de laboratorio en los procesos de enseñanza y en el aprendizaje significativo de las ciencias experimentales por parte de los estudiantes.
A partir de la última década se han adelantado investigaciones sobre $\mathrm{PL}$, que permiten renovar los trabajos prácticos tradicionales (Caamaño - Carrascosa y Onorbe 1994; Hodson 1994) y han generado un amplio consenso en torno a su orientación como una actividad investigativa (González, 1992; Grau, 1994; Lillo. 1994; Watson, 1994). Estas suponen el tratamiento de situaciones problemáticas de interés y exigen verdaderos programas de investigación conocidos y orientados por el profesor. De esta manera se favorece la construcción de conocimientos y una modificación frente a la visión de ciencia y del trabajo científico por parte de la comunidad de profesores en formación de ciencias. 


\section{Aspectos METOdológicos}

Para la recolección de la información se utilizaron encuestas aplicadas a profesores y estudiantes, los portafolios de cada uno de los profesores, los informes de laboratorio elaborados por los estudiantes, el análisis de guías elaboradas por los docentes, entrevistas efectuadas a profesores y estudiantes, y la filmación de las practicas de laboratorio realizadas.

La muestra poblacional estuvo constituida por (92) estudiantes de los grupos de Teorías Químicas I al IV; y por (6) profesores que orientas los cursos de química en el ciclo de fundamentacion.

\section{Resultados}

\section{Encuestas aplicadas a profesores y estudiantes}

De acuerdo a las encuestas aplicadas se presenta en la Tabla No.1, en la cual se presentan los aspectos más relevantes al realizar prácticas de laboratorio por estudiantes y profesores del proyecto curricular de la Licenciatura en Química.

\section{Análisis de portafolios}

Se manifiesta una tendencia generalizada en la mayoría de los núcleos a solicitarle al estudiante la elaboración de propuestas experimentales asociadas a temáticas específicas. No aparecen en los datos señales claras sobre la labor previa del profesor que permitan que los estudiantes efectivamente propongan actividades experimentales coherentes con un proceso formativo en términos de resolución de problemas. Parece existir en la mayoría de profesores la idea de que el cambio a producir en la realización de
PL se logra en la medida en que los estudiantes son quienes plantean las prácticas a desarrollar.

\section{Informes de laboratorio}

En general, los informes presentados sobre reacción química, periodicidad química y leyes de los gases, responden a un formato tradicional que contemplan aspectos tales como: objetivos, marco teórico, materiales y reactivos, metodología, conclusiones y bibliografía.

\section{Guías dadas por el profesor}

Las guías analizadas pueden ser clasificadas en dos grupos: Guías definidas típicamente como tradicionales pero con algunas modificaciones; y las guías que pretenden responder de alguna manera a la idea de insertar las PL dentro de procesos de resolución de problemas.

\section{Entrevistas a profesores y estudiantes}

Fueron realizadas (5) entrevistas semiestructuradas por medio de las cuales se les solicitaba a los profesores dar a conocer su apreciación respecto al papel del experimento en la producción del conocimiento químico, las orientaciones dadas por los docentes a las prácticas de laboratorio y evaluación.

\section{Filmación de prácticas de}

\section{laboratorio}

Se realizaron (20) filmaciones de las prácticas de laboratorio realizadas en los cursos de teorías químicas. A partir de las filmaciones realizadas, se realizo la respectiva trascripción con el fin de establecer patrones o criterios comunes que se detectaran en ellas. 
Tabla No. 1 Aspectos relevantes al realizar practicas de laboratorio

\begin{tabular}{|c|c|c|c|c|}
\hline $\begin{array}{l}\text { Aspectos referidos a las } \\
\text { practicas de laboratorio }\end{array}$ & \multicolumn{2}{|c|}{$\begin{array}{c}\text { Respuestas dadas por } \\
\text { los estudiantes } \\
N=92\end{array}$} & \multicolumn{2}{|c|}{$\begin{array}{c}\text { Respuestas dadas por } \\
\text { los profesores } \\
N=8\end{array}$} \\
\hline Importancia dada a la $P L$ & $\begin{array}{c}\text { Comprobación } \\
\text { de la teoría } \\
(67 \%)\end{array}$ & $\begin{array}{c}\text { Elaborar } \\
\text { guias }(23 \%) \\
\text { Desarrollo de } \\
\text { habilidades y } \\
\text { destrezas }(10 \%\end{array}$ & $\begin{array}{l}\text { Comprobación } \\
\text { de teorías } 75 \%\end{array}$ & $\begin{array}{c}\text { Formación } \\
\text { investigativa } \\
25 \%\end{array}$ \\
\hline Objetivos que se persiguen & $\begin{array}{c}\text { Comprobación } \\
\text { teórica de las } \\
\text { temáticas vistas } \\
(64 \%)\end{array}$ & $\begin{array}{c}\text { Desarrollo de } \\
\text { habilidades } \\
\text { y destrezas } \\
(35 \%)\end{array}$ & $\begin{array}{l}\text { Comprobación } \\
\text { de teorias } 75 \%\end{array}$ & $\begin{array}{c}\text { Desarrollo de } \\
\text { habilidades } \\
\text { y destrezas } \\
25 \%\end{array}$ \\
\hline Orientación & \multicolumn{2}{|c|}{$\begin{array}{l}\text { A través de guías }(70 \%) \\
\text { Explicación metodología } \\
\text { al inicio del laboratorio }(27 \%)\end{array}$} & \multicolumn{2}{|c|}{$\begin{array}{l}\text { A través de guías elaboradas } \\
\text { por los estudiantes }(95 \%)\end{array}$} \\
\hline Formas de Evaluación & \multicolumn{2}{|c|}{$\begin{array}{l}\text { Informes luego de efectuada } \\
\text { la practica }(80 \%)\end{array}$} & \multicolumn{2}{|c|}{$\begin{array}{l}\text { Informes }(60 \%) \\
\text { Preinformes }(15 \%) \\
\text { Quizzes }(15 \%)\end{array}$} \\
\hline $\begin{array}{c}\text { Critica a las guias de } \\
\text { laboratorio }\end{array}$ & $\begin{array}{l}\text { Deben ser } \\
\text { elaboradas por } \\
\text { el estudiante } \\
(57 \%)\end{array}$ & $\begin{array}{l}\text { Guias } \\
\text { repetitivas } \\
\text { extraídas } \\
\text { de textos } \\
(42 \%)\end{array}$ & $\begin{array}{l}\text { No deben } \\
\text { ser de corte } \\
\text { tradicional } \\
(77 \%)\end{array}$ & $\begin{array}{c}\text { Planteadas } \\
\text { desde una } \\
\text { perspectiva } \\
\text { investigativa } \\
(20 \%)\end{array}$ \\
\hline
\end{tabular}

\section{Conclusiones}

Sobre las concepciones y acciones de los profesores acerca de las prácticas de laboratorio:

1. La mayoría de los profesores de química consideran importante, para los procesos de enseñanza y aprendizaje de esta ciencia, la realización de prácticas de laboratorio ya que son un medio propicio para la comprobación de las teorías vistas en el aula de clase y para el desarrollo de habilidades y destrezas en el reconocimiento, manejo y utilización de los equipos, materiales y reactivos. Sin embargo, el tiempo que se dedica a la realización de prácticas de laboratorio es escaso y no necesariamente se establece una coherente relación entre su planteamiento y ejecución con estructuras teóricas debidamente articuladas. Se trata más bien, de actividades puntuales referidas a ciertos tópicos que en su conjunto no responden a cuerpos conceptuales articulados. No aparecen referencias explícitas a utilizar las Prácticas de Laboratorio como medio para la familiarización del estudiante con las características propias del trabajo científico, ni como medio para favorecer aprendizajes significativos de la química. 
2. Cuando los profesores son puestos en situación de analizar críticamente una guía tradicional, la mayoría, se refiere negativamente a la estructura lineal rígida de la misma ya que presenta de forma secuencial los objetivos, las reacciones químicas, las gráficas de los montajes, el listado de materiales y reactivos y los pasos a seguir. Son muy pocos los profesores que en su crítica refieren a la ausencia de situaciones problemáticas y a la posibilidad de generar hipótesis durante el proceso de resolución de problemas. En el análisis no aparecen sugerencias concretas de los profesores para mejorar la calidad de la guía suministrada.

3. Parece existir en la mayoría de profesores la idea, que el cambio a producir en la realización de Prácticas de Laboratorio se logra si los estudiantes son quienes plantean las prácticas a desarrollar proponiendo sus propias guías. Esta es una situación que consideramos muy delicada respecto a la formación científica de los estudiantes puesto que no se proveen las condiciones para que ellos vivencien, con la orientación del profesor, los momentos más importantes vividos por quienes investigan, será muy difícil para los estudiantes realizar lo que no conocen.

\section{Los profesores orientan las Prácti-} cas de Laboratorio mediante guías que ellos mismos elaboran o que son propuestas por los estudiantes. El análisis de las guías suministradas por los profesores permite clasificarlas en dos grupos. En el primer grupo están las guías definidas típicamente como tradicionales pero con algunas modificaciones; en el segundo, aquellas que pretenden responder de alguna manera a la idea de insertar las Prácticas de Laboratorio dentro de procesos de resolución de problemas. Las guías tradicionales presentan un formato que hace énfasis en el procedimiento a seguir acompañado, la mayoría de las veces, con gráficos para los montajes y/o con tablas para la toma de datos por parte de los estudiantes, y los materiales y equipos ha utilizar. En general, no presentan referentes teóricos y las tareas asignadas al finalizar la guía, obedecen más a aspectos particulares de la misma práctica que a cuestiones conceptuales básicas. En el otro grupo de guías, se encuentran aquellas que de alguna manera refieren las Prácticas de Laboratorio a procesos de resolución de problemas. Dicha referencia se reduce a solicitarle al estudiante que "Escriba la situación problema que se describe en la práctica No. X". No se hace explícita la orientación para que los estudiantes analicen y acoten el problema, emitan las hipótesis, diseñen el experimento, obtengan resultados, analicen y concluyan dentro de las teorías estudiadas y mucho menos formulen nuevas situaciones problemáticas que generen nuevas opciones de trabajo experimental.

5. La evaluación del trabajo realizado por los estudiantes cuando realizan prácticas de laboratorio se realiza fundamentalmente con base en los informes presentados por los estudiantes después de finalizadas. En menor proporción son tenidas en cuenta actividades tales como: discusión en el aula sobre la práctica efectuada, análisis de resultados, desempeño individual y grupal, manejo de material y consultas sobre el tema. No existen referencias a la evaluación de aspectos tales como: formulación e identificación de problemas, identificación de variables, emisión 
de hipótesis, diseño de la práctica de laboratorio, obtención y procesamiento de información y formulación de nuevos problemas.

6. Al analizar los portafolios que los profesores entregan a la dirección del departamento, como parte de la planeación de su trabajo, se puede afirmar que en los espacios académicos se hacen propuestas de trabajo en el laboratorio. Al revisar el contenido de las actividades propuestas en los distintos núcleos se puede concluir que responden a actividades tradicionales que inducen al estudiante a reproducir un conjunto de situaciones tomadas de los libros o de consultas realizadas a través de Internet. Los documentos no aportan datos que permitan caracterizar el trabajo previo del profesor para que los estudiantes efectivamente propongan actividades experimentales coherentes con un proceso formativo en términos de resolución de problemas. En ninguno de los casos, las Prácticas de Laboratorio sugeridas se plantean desde una perspectiva negativa; es decir, no responden a situaciones problemáticas previamente definidas por los profesores.

7. El análisis de los informes de laboratorio presentado por los estudiantes después de finalizar las prácticas permite afirmar que mantienen una estructura en términos de: objetivos, marco teórico, materiales y reactivos, metodología, conclusiones y bibliografía; un buen porcentaje no presenta resultados. Tanto el marco teórico como las conclusiones se limitan a la reproducción de la información suministrada en diferentes libros de texto; no se ofrece un reelaboración de dicha información por parte del estudiante. Si bien, en algunos informes se alude a las hipótesis, su contenido no hace explícitas las va- riables comprometidas en el fenómeno estudiado y por consiguiente, no se presentan diseños experimentales acordes con las mismas. Los procedimientos se limitan a la copia de los pasos seguidos en el laboratorio. En los informes que refieren análisis de resultados, en la mayoría de los casos, se limita a la repetición de los datos sin que exista una relación con la(s) hipótesis y los fundamentos teóricos. No aparecen referencias a situaciones problemáticas a partir de las cuales se realiza el trabajo experimental.

8. En general, las preocupaciones de los profesores respecto al trabajo experimental, expresadas en las entrevistas, no aportan mayor información sobre los principales problemas en este campo. Así, se refieren a aspectos tales como: aumentar el tiempo para la realización de Prácticas de Laboratorio, adquirir equipos más sofisticados para realizar investigación, comprar nuevos reactivos, adecuar espacios físicos, manejar apropiadamente los residuos, no sintetizar algunos elementos químicos.

Al respecto surge la pregunta $¿ S i$ en este momento se diera respuesta positiva a todas estas inquietudes, se solucionaría la problemática de las Prácticas de Laboratorio en la formación de los profesores de química? Desde nuestra perspectiva consideramos que el problema central esta referido a la concepción que se tenga del trabajo científico en química y al papel que en él juega el trabajo experimental. Consideramos que este es un punto absolutamente fundamental, pues de nada valdría la inversión en equipos, reactivos, materiales, planta física, si no se logra un cambio en las concepciones y en las acciones sobre el papel que juegan las Prácticas de laboratorio en la formación científica de los futuros profesores de Química. 
9. El estudio del material obtenido a través de las filmaciones en video permite reconocer un cierto patrón común para la realización de las prácticas de laboratorio. Las actividades en su conjunto llevan a establecer una cierta mecánica que se cumple para todas las sesiones de todas las materias. La preparación previa consiste en la elaboración de un preinforme o en el estudio de la guía entregada por el profesor. El preinforme se corresponde con la guía tradicional.

Las guías que entregan los profesores se corresponden con los preinformes de los estudiantes. En todos los casos esta preparación previa, esta fundamentalmente dirigida a la determinación de los procedimientos a seguir junto con algunas explicaciones adicionales para una mejor compresión. En este trabajo no se advierte con claridad que los estudiantes sean puestos frente a situaciones problemáticas para cuya comprensión y resolución requieran del diseño y ejecución de experimentos que los lleven a plantear las prácticas como espacios de contrastación de sus hipótesis.

En el laboratorio, los estudiantes, en general, se limitan a seguir los pasos descritos en las guías o en los preinformes con el fin de obtener una serie de datos que recogen de manera no sistemática, en hojas sueltas, para su posterior utilización en el informe que deben entregar. En estos procesos se evidencia dificultades en el manejo de materiales y reactivos, en el montaje de los aparatos y en general, en la comprensión teórica de lo que están realizando. En este contexto la labor del profesor se limita a dar algunas instrucciones según las necesidades de los estudiantes, a resolver algunas preguntas y a prestar el apoyo que requieren. Así, las acciones del profesor no se di- rigen a cuestionar al estudiante respecto a aspectos fundamentales del trabajo científico tales como: el reconocimiento y control de variables, los aspectos tecnológicos de los diseños experimentales utilizados, la coherencia o no de los resultados obtenidos y el análisis de los mismos en relación con las teorías referidas al fenómeno estudiado. Es decir, No hay una discusión "seria" que favorezca procesos de pensamiento crítico durante la ejecución de las Prácticas de Laboratorio. Pareciera que el gran objetivo del trabajo práctico consiste en obtener unos datos para luego elaborar el correspondiente informe. en una sesión, el profesor refiere el trabajo práctico a situaciones problemáticas, sin embargo, el análisis de las preguntas que plantean el problema nos permite afirmar que realmente no se refieren a situaciones problemáticas; la pregunta refiere al ¿Qué es?, lo cual direcciona el pensamiento del estudiante a responder a través de una definición quedando ya resuelta la cuestión.

10. Los profesores de Química, en general, no hacen mención al papel del experimento en la producción de nuevo conocimiento en este campo. La concepción de experimento la asocian a la realización de prácticas de laboratorio a las cuales asignan una función eminentemente comprobatoria de la teoría. Los resultados obtenidos permiten plantear la preocupación acerca de la formación científica de los profesores de química; podría pensarse en la existencia de una muy escasa relación de los profesores con la investigación científica; esta situación de ser totalmente cierta dificultaría en gran parte la puesta en escena de actividades de laboratorio basadas en la resolución de problemas. Sobre el planteamiento de los estudiantes acerca de las prácticas de laboratorio: 
a. En concordancia con los resultados encontrados para el caso de los profesores, los estudiantes consideran que los docentes atribuyen al trabajo práctico un gran papel como medio para la comprobación de la teoría y en esto radica la importancia de las Prácticas de Laboratorio. Una gran minoría señala que para los profesores es importante utilizar las Prácticas de Laboratorio para que los estudiantes realicen consultas, elaboren guías y mejoren sus habilidades para reconocer y manejar materiales.

b. Existe una opinión generalizada en los estudiantes respecto a que las Prácticas de Laboratorio tienen como finalidad la comprobación teórica de los temas vistos en el aula de clase. También, se reconoce como un objetivo a lograr el desarrollo de habilidades para el manejo adecuado del material de laboratorio. En ningún caso se señala la idea de asociar las prácticas de laboratorio con procesos referidos a la resolución de situaciones problemáticas como medio adecuado para aproximar a los estudiantes al trabajo científico y favorecer el aprendizaje significativo de la Química.

c. La mayoría de los estudiantes consideran que los profesores orientan la realización de las Prácticas de Laboratorio a través de guías previamente entregadas o con explicaciones de la metodología a seguir hechas al iniciar la sesión de laboratorio. Otros señalan que la orientación del profesor se realiza a través de la realización de preinformes que permiten aclarar diferentes aspectos de la práctica. Así, la orientación didáctica realizada por los profesores no está referida con el planteamiento y resolución de situaciones problemáticas d. Los estudiantes al analizar las guías de laboratorio, en su mayoría, plantean que el trabajo práctico debe ser totalmente desarrollado por el estudiante y no mediante guías suministradas por parte del docente. Una buena cantidad de estudiantes hacen alusión a que las guías suministradas por algunos docentes, presentan una información repetitiva la cual se extrae de los textos. Los estudiantes no señalan en sus críticas, la ausencia en las guías de situaciones problemáticas a las cuales responda el trabajo práctico; tampoco se critica la limitación de la guía para que los estudiantes puedan: emitir hipótesis, diseñar y ejecutar el experimento, así como plantear nuevas situaciones problemáticas.

e. La mayoría de los estudiantes señala que la forma más utilizada para evaluar el trabajo de laboratorio es a través de los informes. Afirman que no son evaluados en aspectos tales como: discusión en el aula sobre la práctica efectuada, objetivos alcanzados en la práctica, asistencia, desempeño individual y grupal, manejo de material, consultas sobre el tema. No existen referencias a que la evaluación incluya situaciones relacionadas con el trabajo científico tales como: formulación e identificación de problemas, identificación de variables, emisión de hipótesis, diseño de la práctica de laboratorio, obtención y procesamiento de información y formulación de nuevos problemas.

f. Los estudiantes en su mayoría encuentran conveniente la realización de informes, ya que estos son una herramienta útil que permite clarificar los conceptos teóricos. Otros manifiestan que se debe llevar a cabo otras formas de evaluación para los informes de laboratorio. No aparecen críticas referidas a 
la estructuración de dichos informes en términos del planteamiento y resolución de situaciones problemáticas.

g. En concordancia con la opinión dada por los profesores a través de las entrevistas, los estudiantes no aportan mayor información sobre los principales problemas frente al trabajo experimental. No hacen alusión al papel del experimento en la producción del conocimiento químico, y simplemente, manifiestan una tendencia generalizada a que las prácticas de laboratorio son el medio para contrastar y corroborar teorías vistas en el aula de clase.

h. En el laboratorio, los estudiantes en general, se ocupan en seguir los pasos descritos en las guías o en los preinformes con el fin de obtener una serie de datos que recogen de manera no sistemática, en hojas sueltas, para su posterior utilización en el informe que deben entregar. En estos procesos se evidencia dificultades en el manejo de materiales y reactivos, en el montaje de los aparatos y en general, en la comprensión teórica de lo que están realizando.

\section{REFERENCIAS BIBLIOGRÁFICAS}

CAAMAÑO. A, CARRASCOSA. J Y ONORBE. A. 1994. Los trabajos prácticos en las ciencias experimentales. Alambique. Didáctica de las ciencias experimentales. № 2 .

GONZALEZ, E. 1992. ¿Qué hay que renovar en los trabajos prácticos? Enseñanza de las ciencias, 10 (2), $206-211$.

GRAU, R. 1994. ¿Qué es lo que hace difícil una investigación? Alambique. Didáctica de las ciencias experimentales, (2), 27 -35.

HODSON D. 1994. Hacia un enfoque más crítico del trabajo de laboratorio. Revista Enseñanza de las Ciencias. Vol. 12. No. 3, 299 - 313.

LILLO, J. 1994. Los trabajos prácticos de ciencias naturales como actividad reflexiva, crítica y creativa. Didáctica de las ciencias experimentales, 2, 47-56.

PAYA, J. 1991. Los trabajos prácticos en la enseñanza de la Física y la Química. Un análisis crítico y una propuesta fundamentada. Tesis Doctoral. Universidad de Valencia.

SALCEDO L. 1991. Aprendizaje memorístico del Concepto de Equilibrio Químico. VIII Congreso Colombiano de Química Cali, memorias, 4I3-4I5.

SALCEDO, L. 1994. Concepciones de los profesores de Química de secundaria sobre las Prácticas de Laboratorio. IX Congreso Colombiano de Química, Memorias.

WATSON, J. 1994. Diseño y realización de investigaciones en las clases de ciencias. Didáctica de las ciencias experimentales, 2, 57-65. 